\title{
A heterodimer of EsxA and EsxB is involved in sporulation and is secreted by a type VII secretion system in Streptomyces coelicolor
}

\begin{abstract}
Correspondence
Paul Dyson

p.j.dyson@swansea.ac.uk
\end{abstract}

Received 9 December 2009

Revised 5 March 2010

Accepted 8 March 2010

\author{
Sandra Akpe San Roman, ${ }^{1}$ Paul D. Facey, ${ }^{1}$ Lorena Fernandez-Martinez, ${ }^{1}$ \\ Caridad Rodriguez, ${ }^{2}$ Carlos Vallin, ${ }^{2}$ Ricardo Del Sol ${ }^{1}$ and Paul Dyson ${ }^{1}$ \\ ${ }^{1}$ Institute of Life Science, School of Medicine, Swansea University, Singleton Park, \\ Swansea SA2 8PP, UK \\ ${ }^{2}$ Department of Biomedical Research, Center of Pharmaceutical Chemistry, Atabey, Playa, \\ PO Box 6990, Havana, Cuba
}

\begin{abstract}
An esx locus, related to the multiple esx loci of Mycobacterium tuberculosis, is conserved in all sequenced Streptomyces genomes, where it is associated with the developmental regulatory gene $b / d B$. Here we demonstrate that the es $x B A$ operon, comprising part of the locus, has a novel morphogenetic function in the model species Streptomyces coelicolor. This operon encodes two proteins belonging to the WXG-100 superfamily that can form a heterodimer and are secreted in the absence of signal sequences. A mutation in es $x B A$ results in a delay in sporulation, with eventual development of aerial hyphae with chains of abnormally sized spore compartments possessing irregular DNA contents. During early sporulation, expression of the operon is elevated in a b/dB mutant. Other genes in the locus, notably SCO5734 and SCO5721, encode components of a type VII secretion system. Disruption of either of these genes prevents secretion of EsxAB but has no effect on sporulation. To explain the morphogenetic function of EsxAB, we propose that the heterodimer sequesters a regulator of expression of genes involved in nucleoid organization during sporulation.
\end{abstract}

\section{INTRODUCTION}

Gram-positive Streptomyces typically inhabit terrestrial soils and marine sediments as free-living saprophytes. In a similar manner to a filamentous fungus, a streptomycete colonizes its particulate environment by growing branching multigenomic hyphae that form a ramifying network, enabling the organism to exploit a localized nutrient source. Being non-motile, they achieve dispersal by means of unigenomic spores borne on specialized non-feeding aerial hyphae. The latter grow out of the semi-aqueous environment inhabited by the feeding vegetative hyphae of terrestrial streptomycetes and ultimately undergo multiple coordinated cell division, generating chains of spores (Flardh \& Buttner, 2009). The spores can then be dispersed

Abbreviations: AFM, atomic force microscopy; fluo-WGA, fluoresceinconjugated wheatgerm agglutinin; Q RT-PCR, quantitative RT-PCR; $\mathrm{RD} 1$, region of difference 1 .

Two supplementary figures, showing the results of RT-PCR analysis of the esxAB operon and alignments of EsxA and EsxB proteins of Mycobacterium tuberculosis and Streptomyces coelicolor, and three supplementary tables, showing a comparison of proteins encoded by the esx locus of Streptomyces griseus and Streptomyces roseosporus, predicted proteins encoded by the $S$. coelicolor esx cluster aligned with orthologues from other streptomycetes, and oligonucleotides used in the study, are available with the online version of this paper. by physical agents or the activities of motile animals inhabiting the same niche. The programme of morphological development, initiated by growth of aerial hyphae, is controlled by developmental genes that include the bld genes: mutations in these genes can prevent aerial growth. Subsequent maturation of aerial hyphae into mature spore chains, which in the case of the model organism Streptomyces coelicolor acquire a typical grey pigmentation, is dependent on developmental whi genes. The sporulation process depends on multiple synchronised cell division to subdivide a long syncytial aerial compartment into many spore compartments, into each of which a single copy of the linear chromosome is segregated.

Streptomyces are related to other G + C-rich Actinobacteria, including rod-shaped mycobacteria, of which Mycobacterium tuberculosis in particular represents a major global human health problem. A key virulence determinant of this bacterium is the esx-1 locus. This is a highly conserved locus present in several related copies in the genomes of indvidual mycobacteria species (Cole et al., 1998; Gey Van Pittius et al., 2001). A deletion within the esx-1 locus is the primary attenuating mutation of the vaccine strain Mycobacterium bovis BCG (Behr et al., 1999; Mahairas et al., 1996), and the locus is also deleted in other attenuated members of the $M$. tuberculosis complex, including the vole bacillus 
Mycobacterium microti (Brodin et al., 2002). The deleted region (known in M. bovis BCG as region of difference 1; $\mathrm{RD} 1$ ) includes genes encoding EsxA and EsxB (also known as ESAT-6 and CFP-10, respectively), which are small secreted proteins that lack classical signal sequences and which form a heterodimer (Renshaw et al., 2005). Other genes in the locus encode the ESX-1 membrane-bound secretory apparatus, with protein export driven by ATP hydrolysis. For example, both $r v 3870$ and $r v 3871$ encode proteins, EccCa and EccCb, respectively (Bitter et al., 2009), belonging to the FtsKSpoIIIE ATPase family that are required for secretion of EsxA and EsxB (Simeone et al., 2009). The ESX-1 apparatus is the paradigm for bacterial type VII secretion systems (Abdallah et al., 2007).

Genome analyses suggest that a related secretion system is found in other Actinobacteria and in some Firmicutes (Pallen, 2002). For the latter, it is encoded by a conserved eight gene cluster, three of which share homology with elements of a mycobacterial esx locus (Burts et al., 2008). For example, Staphylococcus aureus EsxA and EsxB proteins share homology with their mycobacterial counterparts, and their secretion is dependent on EssC, an FtsK-SpoIIIE ATPase (Burts et al., 2005). Mutants defective in production of EsxA and EsxB exhibit decreased virulence, with the suggestion that the secretion system has a role in promoting persistent infections. The function of the secretion system in other Actinobacteria is unknown. Although Corynebacterium diphtheriae contains a single orthologous locus (Gey Van Pittius et al., 2001), the gene cluster is not confined to pathogens. For example, the genome of free-living $S$. coelicolor also contains a single complete gene cluster containing orthologues of the typical mycobacterial esx locus (Gey Van Pittius et al., 2001). Here we demonstrate that SCO5725 (EsxA) and SCO5724 $($ EsxB) in S. coelicolor are novel morphogenetic proteins whose secretion is dependent on functions encoded by at least two other genes in the locus.

\section{METHODS}

Bacterial strains and media. S. coelicolor A3(2) and Escherichia coli strains are listed and described in Table 1. Cloning procedures were performed in E. coli JM109, while E. coli ET12567/pUZ8002 was used for intergeneric conjugative transfer of plasmid DNA into Streptomyces strains (Kieser et al., 2000). Culturing of E. coli strains was as recommended by Sambrook et al. (1989). S. coelicolor strains were grown on the surface of MS (mannitol soya flour), R2YE or NMMP (no additional carbon source added) agar (Kieser et al., 2000) or in submerged culture with Tryptone Soy Broth (Lab M). Streptomyces mutant strains (Table 1) were obtained using Tn5062mutagenized cosmids (Bishop et al., 2004). In the case of the bldB mutant DSCO5723, no insertions in this small gene had been identified in the original insertion library. Consequently, plasmid pSA11 was subjected to in vitro transposon mutagenesis as described by Bishop et al. (2004). Transposon insertions were mapped by DNA sequencing, and a transposant carrying an insertion in the bldBcoding sequence at chromosomal position 6244029 (tnpSA11-F11) was conjugated into $S$. coelicolor M145. Exconjugants carrying a chromosomal copy of the disrupted version of $b l d B$ were selected. The identity of all mutants was confirmed by Southern blotting (Sambrook et al., 1989).

DNA manipulation and plasmid construction. Standard DNA procedures were performed as described by Sambrook et al. (1989). Plasmid pSA1 was obtained by digesting pBlueScript SK II + with NotI and ligating with a NotI fragment from cosmid SC3C3.1.H04 containing the apramycin-resistance gene of Tn5062, bldB and es $x B A$. Conveniently located $\mathrm{BamHI}$ sites within the insert and the plasmid backbone permitted removal of all remaining transposon sequences and part of bldB, resulting in pSA2. For complementation analysis, the fragment containing esxAB was subsequently subcloned as a BamHI-EcoRV fragment into the conjugative integrative plasmid pSH152, yielding pSA3. Construction of a C-terminal His-tagged EsxA required the introduction of a $\mathrm{XhoI}$ site replacing the es $x A$ stop codon in pSA2 by site-directed mutagenesis using oligonucleotides EsatMutEnd1 and -2 (Supplementary Table S3), resulting in plasmid pSAXhoI. This plasmid was digested with BamHI and XhoI, and the fragment containing es $x A B$ was recovered and combined with a XhoISmaI fragment containing the His-tag-coding sequence released from pET26b. The ligated fragment was cloned into pSH152 previously digested with BamHI and EcoRV, yielding pSAHis2. Similarly, an es $x B_{\text {His } 6}$ fusion was constructed by introducing a $\mathrm{XhoI}$ site replacing the $e s \times B$ stop codon by site-directed mutagenesis (oligonucleotides CFPMutEnd 1 and -2 ) on plasmid pSA2, resulting in pSA2m3. The latter was digested with EcoRV/XhoI and, together with the XhoISmaI fragment from pET26b mentioned above, ligated to pSH152 digested with EcoRV. The resulting integrative plasmid encoding es $x B_{H i s 6}$ was called pSAHis4.

For mutagenesis of bldB, plasmid pSA1 was digested with HindIII and the $2426 \mathrm{bp}$ insert subcloned into pCR2.1-TOPO (Invitrogen) to produce plasmid pSA11. Plasmids for two-hybrid studies were constructed as follows. pSA2 was used as a template in combination with oligonucleotide pairs CFPMUT1 and -2 and ESATMUT1 and -2 (Supplementary Table S3) for consecutive site-directed mutagenesis to introduce $X m a \mathrm{I}$ and $B a m \mathrm{HI}$ sites that overlapped the es $x B$ and es $x A$ start codons, respectively, resulting in plasmid pSA2m2. This plasmid was digested with XmaI, BamHI and XhoI, and the XmaI-BamHI $e s \times B$ and $B a m \mathrm{HI}-X h o \mathrm{I} e s x A$ fragments were recovered and ligated to, respectively, XmaI/BamHI-digested $\mathrm{pBT}$ and pTRG digested with $B a m \mathrm{HI}$ and XhoI, producing plasmids pBTCFP and pTRGEST. To switch bait and target protein fusions, the es $x A$ fragment in pTRGEST was excised with NotI and XhoI and ligated to $\mathrm{pBT}$ digested with the same pair of restriction enzymes to produce pBTEST. Likewise, the es $x B$ fragment in pBTCFP was excised with BamHI and XhoI and ligated with pTRG cut with the same enzymes to produce pTRGCFP. Total DNA from $M$. tuberculosis $\mathrm{H} 37 \mathrm{Rv}$ was used as template for the PCR amplification of $e s x B_{M t b}$ and $e s x A_{M t b}$ using the oligonucleotide primer pairs TbCFPF1/TBCFPR1 and TbESATF1/TbESATR1 (Supplementary Table S3). The resulting PCR products were digested with EcoRI and BamHI and cloned into pBT and pTRG plasmids digested with the same enzyme combination to produce pBTbesxB and pTTbesxA.

RNA isolation and quantitative RT-PCR (Q RT-PCR). Total RNA isolation and RT-PCR/Q RT-PCR procedures were performed as previously described (Del Sol et al., 2006; Fernandez-Martinez et al., 2009). Briefly, sterile cellophane disks placed on top of MS plates were inoculated with Streptomyces spores. Disks remained in place for a specified time, after which mycelia were collected and total RNA was isolated with a Qiagen RNeasy Mini kit. cDNAs were obtained using a RETROscript reverse transcription kit (Ambion) with random decamers. Q RT-PCR was carried out on an iCycler iQ real-time PCR detection system (Bio-Rad) using SYBR Green Supermix $2 \times$ containing Thermo-Start DNA polymerase (ABgene). Gene-specific primers (Supplementary Table S3) were designed using Beacon 
Table 1. Bacterial strains and plasmids

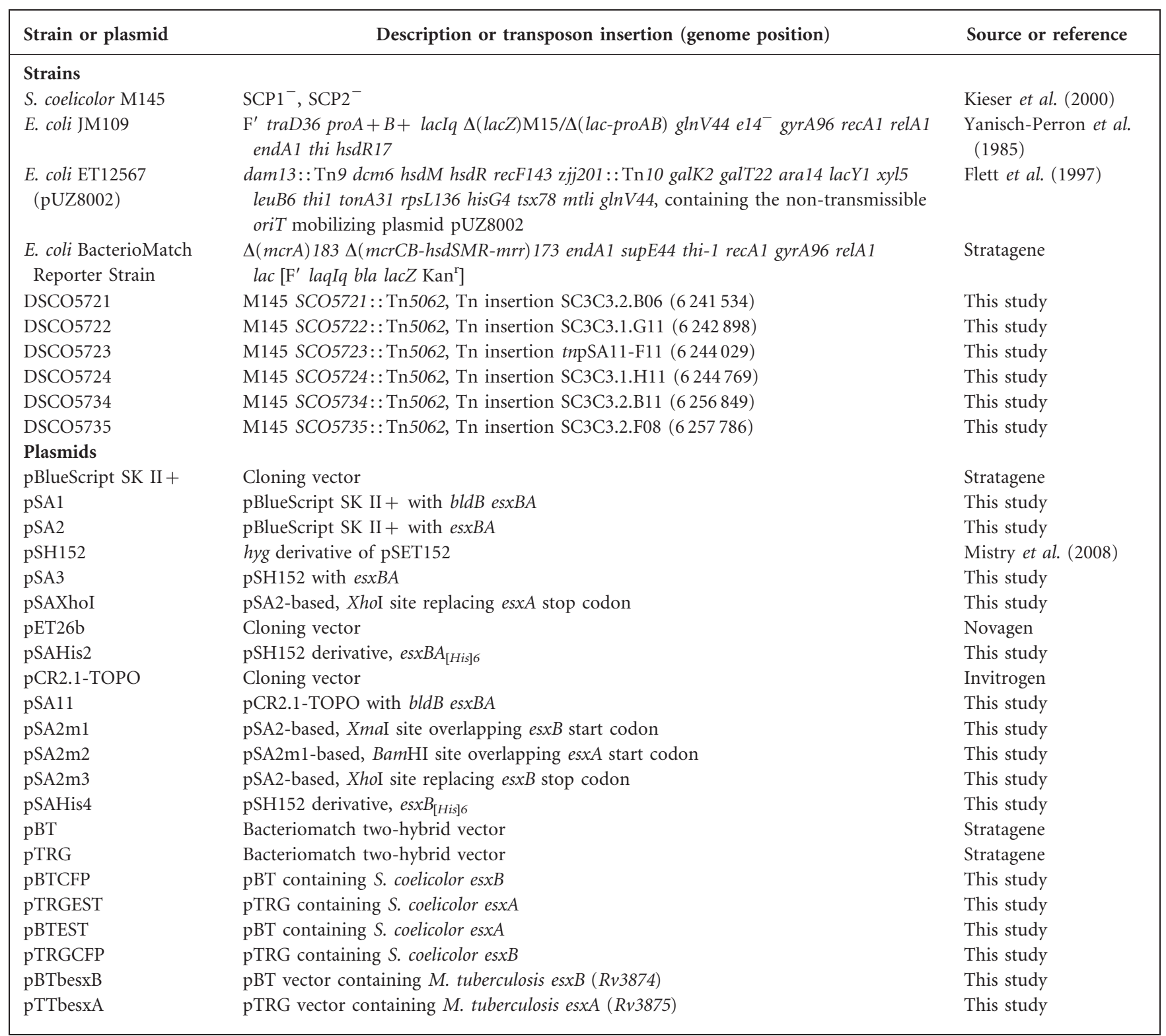

Design 2.0 (Premier Biosoft). Dilutions of S. coelicolor genomic DNA of known concentration were used as standards and total RNA samples were included as negative controls. The S. coelicolor hrdB gene, encoding the principal sigma factor, was used as an internal control.

Protein methods. Strains carrying pSAHis2 were grown in Tryptone Soy Broth until mid-exponential phase (around $24 \mathrm{~h}$ at $30{ }^{\circ} \mathrm{C}$, 250 r.p.m.). Complete Protease Inhibitor (Roche) was added to the culture and cells were collected by centrifugation. The supernatant was treated with $2 \%$ TCA at $4{ }^{\circ} \mathrm{C}$ for $1 \mathrm{~h}$, followed by centrifugation at $40000 \mathrm{~g}$ for $20 \mathrm{~min}$. The resulting pellet was washed twice with icecold acetone and left to air dry. Sonication buffer $(50 \mathrm{mM}$ Tris/ $\mathrm{HCl}$, $\mathrm{pH} 8.0,200 \mathrm{mM} \mathrm{NaCl}, 15 \mathrm{mM}$ EDTA) was added to resuspend precipitated proteins and was subsequently mixed with an equal volume of $2 \times$ Laemmli buffer. The protein concentration of the resuspended sample was determined using a 2-D Quant kit (GE Healthcare). Intracellular proteins were released by sonication $(20 \mathrm{~s}$ burst on ice) of the cell pellet resuspended in ice-cold sonication buffer. Unbroken cells and insoluble components were removed by centrifugation at $40000 \mathrm{~g}$, and the resulting supernatant was used to assess soluble intracellular proteins. The concentrations of soluble protein fractions were determined using Bradford reagent (Bio-Rad). Protein samples $(5-10 \mu \mathrm{g}$ total protein) were separated on a $12 \%$ SDS-PAGE gel and transferred to Hybond-P (GE Healthcare). Histagged EsxA was detected using a penta-His-peroxidase conjugate antibody (Qiagen) and an ECL Advance Western blotting detection kit (GE Healthcare) following the manufacturer's recommendations.

Bacterial two-hybrid assays. The BacterioMatch II two-hybrid system (Stratagene) was used to explore protein interactions. Translational fusions were constructed and introduced into the supplied E. coli BacterioMatch II two-hybrid system reporter strain. $\beta$-Galactosidase assays were performed as recommended by the manufacturer, and for each interaction tested at least four independent clones were analysed and the combined data then averaged. 
Assessment of mutant phenotypes. To assess colony morphology, strains were plated on MS agar (Kieser et al. 2000). Strains used in fluorescent microscopy were taken as coverslip impressions on the surface of MS growing colonies after 3 days or grown in the acute angle of a coverslip inserted in the agar medium. Cell walls and DNA were stained with fluorescein-conjugated wheatgerm agglutinin (fluoWGA) and propidium iodide (both from Molecular Probes), respectively, as described by Schwedock et al. (1997). Live-cell nucleoids were stained with SYTO9 (Invitrogen) (5 $\mu \mathrm{M}$ diluted in $20 \%$, v/v, glycerol). Images were obtained using a Nikon Eclipse E600 epifluorescence microscope fitted with a CoolSNAP microscope camera (RS Photometrics). Fluorescence intensity profiles along spore chains stained with SYTO9 were created in ImageJ (Abramoff et al., 2004). Briefly, freehand lines, approximately $12 \mu \mathrm{m}$ in length, were drawn along the spore chains, passing through the centre of adjacent nucleoids. Fluorescent profiles were created using the 'plot profile' function. Measurements of spore compartment size were performed using Scion Image software (Scion Corp.); at least 500 measurements per strain were performed. Atomic force microscopy (AFM) samples were prepared and imaged as described previously (Del Sol et al., 2007).

\section{RESULTS}

\section{Architecture of the conserved esx locus in Streptomyces}

Analysis of sequenced streptomycete genomes for the presence of the minimal esx cluster originally identified in S. coelicolor (Gey Van Pittius et al., 2001) revealed a single conserved cluster in five other species (Fig. 1). Certain aspects of the overall architecture of the streptomycete es $x$ locus are conserved. In each case the cluster is delimited on one side by two genes that are likely to be co-transcribed and which encode (i) an N-terminal transmembrane protein with an ATP/GTP-binding site, and (ii) a protein related to the mycosin, subtilisin-like cell wall-associated serine protease family. At the other end of the cluster are two divergently transcribed genes that encode (i) an integral membrane protein and (ii) an FtsK-SpoIIIE ATPase. Contained within each cluster is an orthologue of the es $x B A$ operon, each gene encoding a protein that belongs to the WXG-100 protein family, members of which are characterized by a central amino acid motif Trp-X-Gly and a size of approximately 100 amino acids (Pallen, 2002). For each species the cluster is linked with the bldB gene, which encodes a transcriptional regulator required for aerial development in S. coelicolor (Mishig-Ochiriin et al., 2003; Pope et al., 1998). Whereas the Streptomyces pristinaespiralis genome contains a minimal uninterrupted locus, for the other species additional ORFs separate different elements of the locus. The overall architecture of the Streptomyces roseosporus esx locus resembles that of Streptomyces griseus and the relatedness of these two species is underlined by the very high degree of identity of the proteins encoded by both clusters (Supplementary Table S1). Alignments of the proteins encoded by the S. coelicolor cluster with those of the other species revealed in each case

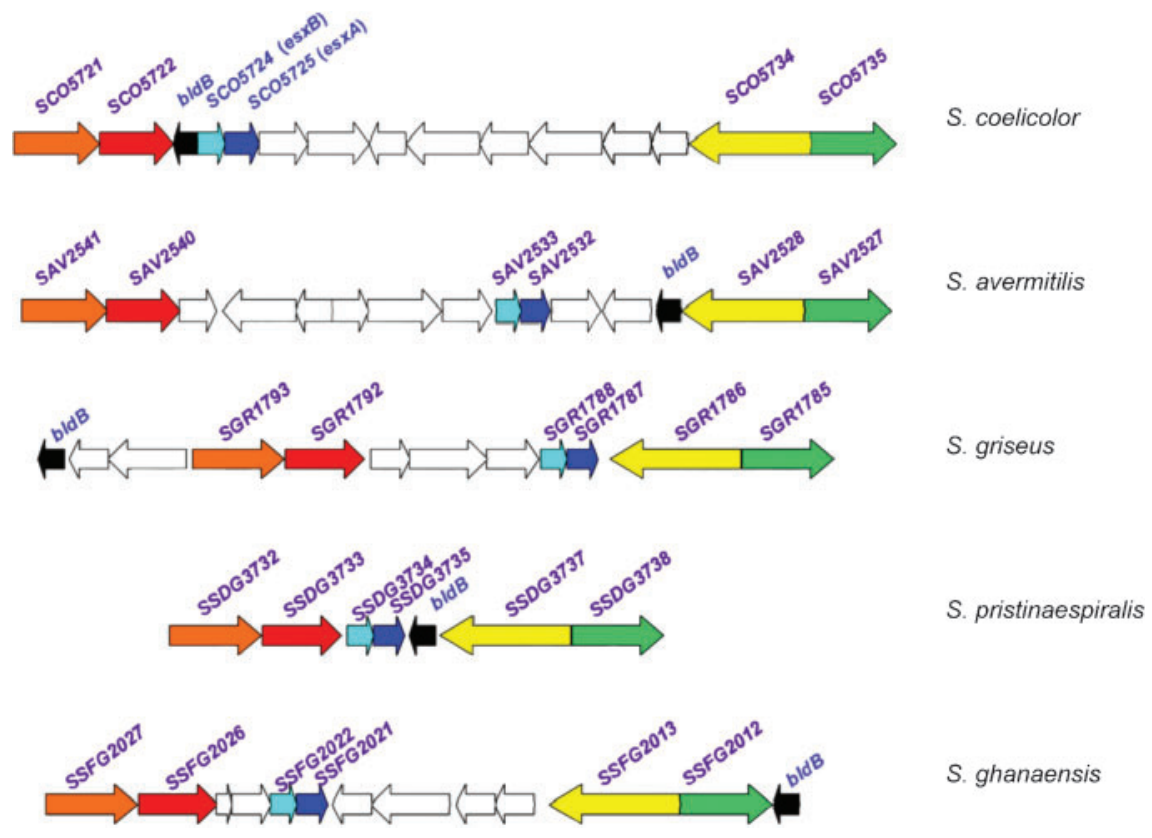

Fig. 1. Architecture of the conserved minimal esx locus in Streptomyces. The genes comprising the esx cluster of $S$. coelicolor (Gey Van Pittius et al., 2001) were compared with their orthologues in five other sequenced streptomycetes. The locus of $S$. roseosporus (not shown) resembles that of $S$. griseus. The colours of genes indicate the corresponding predicted proteins: $\mathrm{N}$-terminal transmembrane protein with ATP/GTP-binding site, orange; subtilisin-like cell wall-associated serine protease, red; BldB regulatory protein, black; EsxB, light blue; EsxA, blue; FtsK-SpollIE ATPase, yellow; integral membrane protein, green. 
that the FtsK-SpoIIIE ATPase is the best-conserved component, whereas the two WXG-100 proteins are generally the least well conserved (Supplementary Table S2). As noted elsewhere (Gey Van Pittius et al., 2001), the locus may extend to include SCO5717 (and the equivalent orthologues in the other five streptomycete genomes), an orthologue of espI ( $r v 3876)$ that is unique to the esx-1 locus of M. tuberculosis.

\section{Disruption of esxBA delays sporulation and affects spore DNA content and morphology}

The es $x B$ and es $x A$ genes are separated by a short $37 \mathrm{bp}$ intergenic region, suggesting their coexpression as an operon. This was confirmed by RT-PCR using as forward primer a sequence from the $5^{\prime}$ region of es $x B$ (oligonucleotide All3S1, Supplementary Table S3) and as reverse primer a sequence complementary to the $3^{\prime}$ sequence of es $x A$ (All2SA1, Supplementary Table S3). The expected-size 715 bp amplicon was only obtained when reverse transcriptase was included in the reaction (Supplementary Fig. S1a). esxA is separated from the next ORF (SCO5726) by a 120 bp intergenic region. No amplicon was obtained after RT-PCR using the same forward primer and a reverse primer complementary to the $3^{\prime}$ sequence of SCO5726 (All3SA1, Supplementary Table S3), although the expected $1.3 \mathrm{~kb}$ amplicon was obtained using chromosomal DNA as template (Supplementary Fig S1b). Hence we conclude that $S C O 5726$ is transcribed independently from the es $x B A$ operon.

To investigate the function of the $S$. coelicolor EsxA and EsxB proteins, a polar mutant, DSCO5724, with a Tn5062 insertion in es $x B$ was constructed. The polarity was confirmed by RT-PCR: using the $5^{\prime}$ es $x B$ forward primer and $3^{\prime}$ es $x A$ reverse primer, an expected $4.2 \mathrm{~kb}$ amplicon was obtained with mutant genomic DNA as template, but not with mutant RNA (results not shown). To address the question of whether esxA is transcribed independently from a second promoter within the operon, another RTPCR was performed with a forward primer complementary to the $5^{\prime}$ region of esxA (EsxAFOR, Supplementary Table S3) and the $3^{\prime}$ es $x A$ reverse primer. With RNA from M145, an expected $194 \mathrm{bp}$ amplicon was obtained, but no amplicon was detected with template RNA from the mutant DSCO5724 (results not shown), suggesting that, with the growth conditions employed, there was no evidence for an es $x A$-specific promoter. Compared with the wild-type M145 strain, the mutant exhibited delayed sporulation on MS medium (Fig. 2a). Whereas M145 sporulated after $36 \mathrm{~h}$ incubation, the mutant required an extra 2-3 days' incubation to reach an equivalent developmental maturity. A delay in sporulation was also evident on several other media tested, including R2YE and NMMP agar (results not shown). To link this developmental phenotype with the esxBA operon, a mutant with an insertion in the immediate downstream gene, SCO5726, was analysed. This mutant underwent sporulation within the same incubation period as the wild-type (data not shown). Introduction of either of the complementing plasmids pSA3 or pSAHis2, each with an intact copy of the es $x B A$ operon, restored the ability of the es $x B A$ mutant to develop normally (Fig. 2a).

The developmental phenotype exhibited by the esxBA mutant prompted microscopic analysis of its spore chains. The aerial hyphae were examined after fluo-WGA staining to reveal cell walls defining spore compartments. Compared with the regularity of spore compartments (mean length $1.3 \mu \mathrm{m}$ ) in the aerial hyphae of M145, the mutant possessed a significantly higher proportion of large compartments of length $>1.9 \mu \mathrm{m}$ (Fig. 2b, Table 2). Live DNA staining of septated aerial hyphae indicated that the aberrant-sized compartments of the mutants correlated with irregular DNA content (Fig. 3). The large compartments of the esxBA mutant appeared to contain multiple nucleoids. While these aberrant compartment sizes with irregular DNA contents are indicative of a role of the es $x B A$ operon in correct septation, additionally we examined the topography of the surface of the spore chains using AFM. The topographic height images and, in particular, the derived corresponding 3D representations revealed a characteristic abnormal 'wrinkled' morphology of the spore compartments of the esxBA mutant, in contrast to the relatively smooth surface topography of wild-type spore compartments (Fig. 2c). AFM at higher-level magnification revealed a normal rodlet layer on the surface of the mutant (data not shown). Spore chains of mutants with either pSA3 or pSAHis2 complementing plasmids resembled the wild-type M145 strain when examined by either epifluorescence microscopy or AFM (data not shown).

\section{EsxB and EsxA of S. coelicolor form a complex}

An alignment of $S$. coelicolor EsxA with EsxA of $M$. tuberculosis revealed $13 \%$ identity and $29 \%$ similarity, and alignment of the two EsxB proteins showed $12 \%$ identity and $34 \%$ similarity (Supplementary Fig. S2). In addition to the conserved WXG motifs, amino acid residues believed to stabilize a mini-hydrophobic core within $M$. tuberculosis EsxA are well conserved (Lightbody et al., 2008). In addition, there is good conservation of critical hydrophobic residues within the $\alpha$-helices that are implicated as lying at intra- and intermolecular interfaces of the fourhelix bundle structure formed by the complex of $M$. tuberculosis EsxA and EsxB (Lightbody et al., 2008).

Based on these alignments it could be expected that, similar to M. tuberculosis EsxA and EsxB, the equivalent $S$. coelicolor proteins would form a $1: 1$ heterodimeric complex. To test this we employed a bacterial two-hybrid system (Karimova et al., 1998), creating translational fusions between the bait protein and $\lambda$ repressor, and the target protein and the $\mathrm{N}$-terminal domain of RNA polymerase. With this system a strong interaction between M. tuberculosis EsxA and EsxB was confirmed, and an 
(a)

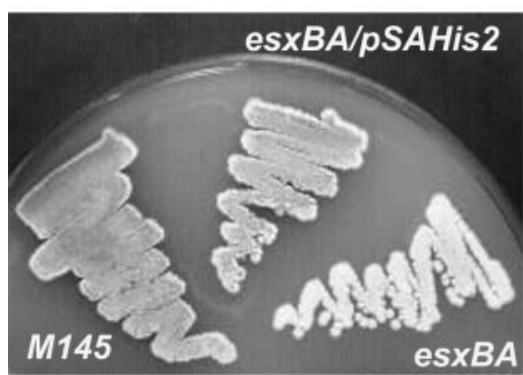

(c)
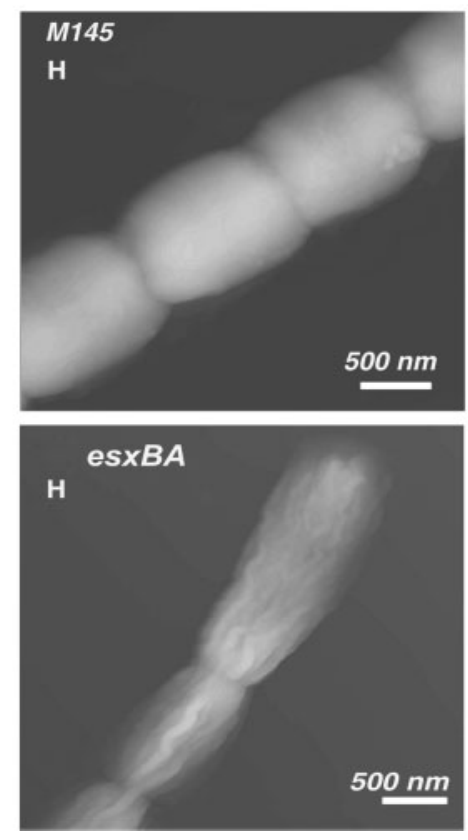

(b)
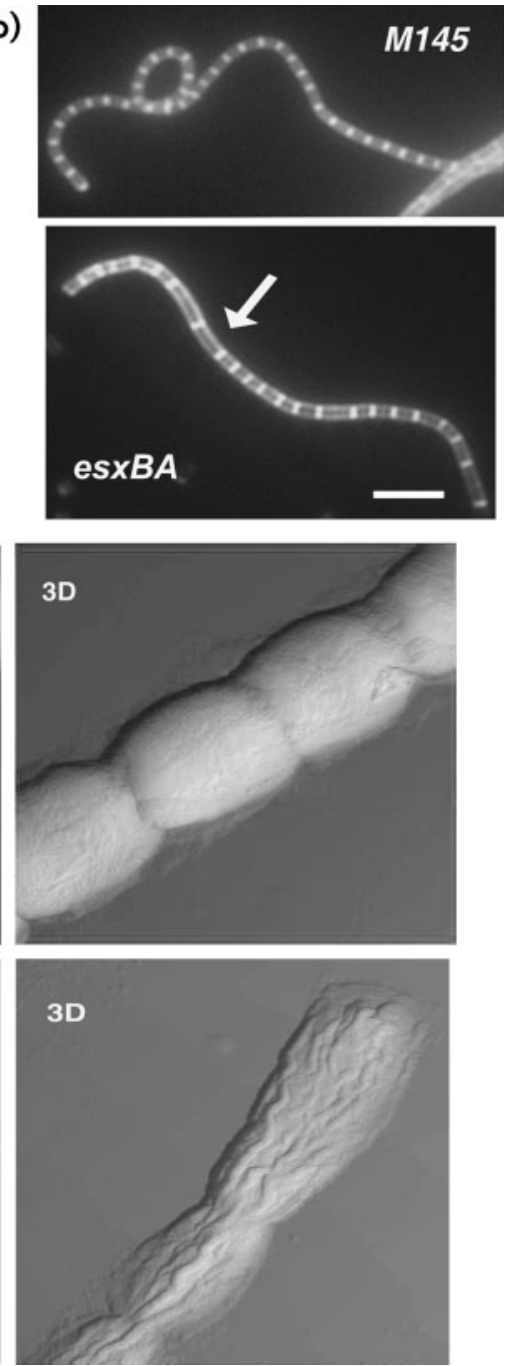

Fig. 2. Delayed sporulation and aberrant spore compartments due to disruption of es $x B A$. (a) A polar es $x B A$ insertion mutant exhibits delayed sporulation compared with the parental M145 strain grown on MS medium for $36 \mathrm{~h}$. (b) Aerial hyphae from $72 \mathrm{~h}$ cultures were stained with Fluo-WGA to reveal sporulation septa; the arrow points to an unusually long spore compartment in the esxBA mutant (bar, $5 \mu \mathrm{m}$ ). (c) AFM topographic height images $(\mathrm{H})$ and corresponding $3 \mathrm{D}$ representations (3D) of spore chains of the parental strain and mutant revealing a 'wrinkled' morphology of the latter. equivalent binding between $S$. coelicolor EsxA and EsxB was measured (Table 3). Similar results were obtained after switching bait and target protein fusions. We also assessed the specificity of complex formation by combining translational fusions of EsxA and EsxB from the two different Actinobacteria: for these combinations we could not detect any interactions. Moreover, as for M. tuberculosis EsxA and EsxB (Lightbody et al., 2004), we could not detect homodimer formation for S. coelicolor EsxA and EsxB.

\section{The S. coelicolor esx locus encodes a type VII secretion system}

The ESX-1 system of $M$. tuberculosis responsible for secretion of EsxA and EsxB, which lack classical signal sequences, is termed a type VII secretion system (Abdallah et al., 2007). To investigate secretion of S. coelicolor EsxA, an additional copy of the operon under the control of the native promoter but expressing a $\mathrm{His}_{6}$-tagged version of the protein was expressed in M145. In a time-course experiment, EsxA[His $]_{6}$ was detected in both culture supernatants and cytoplasmic fractions after $16 \mathrm{~h}$ incubation, reaching maximum abundance after $24 \mathrm{~h}$ but then declining, so that very little could be detected after $40 \mathrm{~h}$ incubation (Fig. 4a). A similar expression profile was observed when a tagged version of es $x B$ controlled by the native promoter was introduced into M145: EsxB[His] 6 was detected in the supernatant of $24 \mathrm{~h}$ cultures, but little or no protein was detected at $40 \mathrm{~h}$ (Fig. 4a). Based on this result, we then examined localization of EsxA[His] $]_{6}$ by expressing an additional copy of the operon in different genetic backgrounds after $24 \mathrm{~h}$ incubation. EsxA[His] $]_{6}$ was detected in the culture supernatants of three mutants with the integrated tagged operon: DSCO5724 with pSAHis2, the complemented esxBA mutant, DSCO5722 with pSAHis2, carrying an insertion in a predicted serine protease gene SCO5722 (Fig. 4b), and DSCO5735 with pSAHis2, disrupted in the integral membrane protein gene SCO5735 (Fig. 4c). However, EsxA[His] $]_{6}$ was not detected in the culture supernatants of either DSCO5734 or DSCO5721 containing pSAHis2, mutants with insertions 
Table 2. Distribution of pre-spore compartment sizes

\begin{tabular}{|lccc|}
\hline \multirow{2}{*}{ Strain } & \multicolumn{3}{c|}{ Percentage of spores of size: } \\
\cline { 2 - 4 } & $<\mathbf{0 . 8} \boldsymbol{\mu m}$ & $\mathbf{0 . 8}-\mathbf{1 . 9} \boldsymbol{\mu m}$ & $>\mathbf{1 . 9} \boldsymbol{\mu m}$ \\
\hline M145 & 0.97 & 95.87 & 3.15 \\
DSCO5724 & 0.4 & 86.16 & 13.43 \\
$($ esxB:: Tn5062) & & & \\
\hline
\end{tabular}

in genes encoding, respectively, an FtsK/SpoIIIE-type protein and an $\mathrm{N}$-terminal transmembrane protein with a likely ATP/GTP-binding domain. EsxA[His] $]_{6}$ was an easily detectable cytoplasmic protein in both these mutants (Fig. 4b). These results indicate that secretion of EsxA is dependent on the activity of two genes in the es $x$ locus that are homologous to genes encoding components of the ESX type VII secretion system of $M$. tuberculosis. Interestingly, apart from the es $x B A$ mutant, none of the other mutants of the $S$. coelicolor ESX-1 locus exhibited delayed sporulation (results not shown).

\section{Expression of the esxBA operon is regulated by BldB}

The operon is divergently transcribed from $b l d B$, a gene known to control aerial development. BldB represses its own expression (Pope et al., 1998) and can bind to a $76 \mathrm{bp}$ region from the $304 \mathrm{bp}$ intergenic region that separates $b l d B$ and esxB (Mishig-Ochiriin et al., 2003). The presence of $b l d B$ within the esx locus prompted investigation of whether $\mathrm{BldB}$ also regulates expression of the es $x B A$ operon. Q RT-PCR of RNA purified at different time points revealed a fourfold increase in expression of es $x B A$ at $36 \mathrm{~h}$ in the $b l d B$ mutant strain DSCO5723 compared with M145 (Fig. 5).
To check whether the developmental phenotype of the es $x B A$ mutant results from an effect of the es $x A B$ mutation on $b l d B$ expression, we performed a time-course analysis of $b l d B$ mRNA abundance, comparing M145 and the esxBA mutant. We found no significant differences between the strains, with increasing abundance observed from $36 \mathrm{~h}$ onwards, in agreement with the expression profile for bldB reported by Pope et al. (1998), indicating that the defect in sporulation and, in particular, nucleoid segregation is indeed due to loss of function of es $x B A$.

\section{DISCUSSION}

We have provided experimental evidence for a type VII secretion system in the model streptomycete $S$. coelicolor. The ESX-1 pathway of M. tuberculosis is the paradigm for these secretion systems in bacteria as, due to its critical role in virulence, it has been the subject of intense analysis. The ESX-1 system has been implicated in innate immune modulation (MacGurn \& Cox, 2007; Stanley et al., 2003, 2007; Volkman et al., 2004), especially early after infection in macrophages, although the precise role of the major secreted substrates and key effector molecules EsxA (ESAT6) and EsxB (CFP-10) is still debated. At least five genes encoded within the approximately 15-gene es $x$ - 1 cluster, $R v 3868$ to $R v 3871$ and $R v 3877$, are required for the secretion of these substrates (Brodin et al., 2006; Guinn et al., 2004; Hsu et al., 2003; Stanley et al., 2003). Rv3877 encodes an integral membrane protein, EccD, that may form all or part of a channel. Rv3869 also encodes a membrane-associated protein. Secretion is believed be driven by ATP hydrolysis: $R v 3870$ and Rv3871 encode ATPases, EccCa and EccCb, respectively, belonging to the FtsK-SpoIIIE family, and Rv3868 encodes another ATPase, EccA.
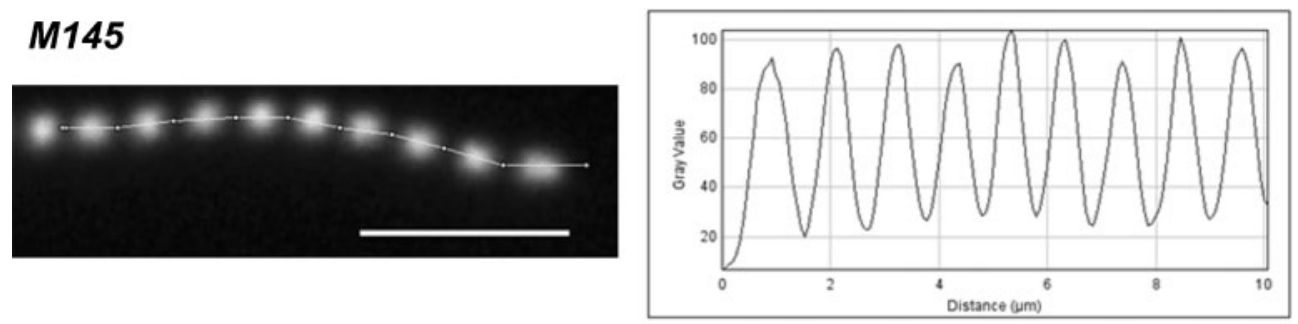

\section{esxBA}
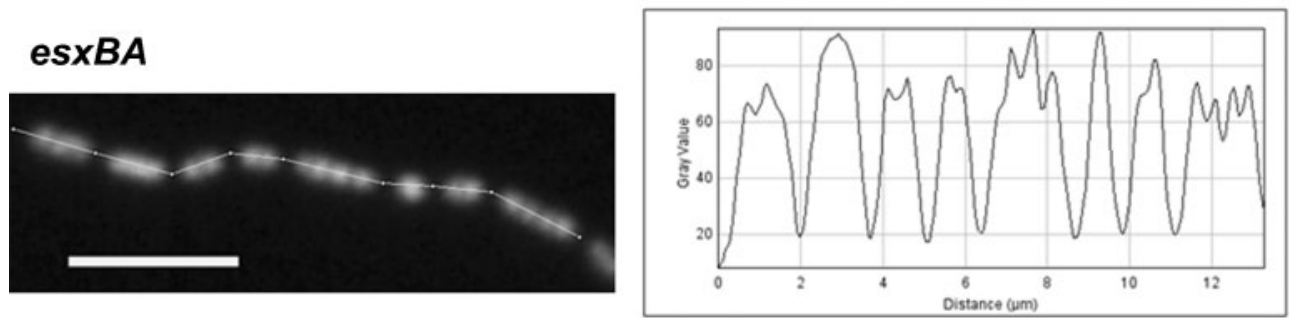

Fig. 3. Fluorescence profiles of SYTO9-stained nucleoids. Images used to determine the profiles are shown next to the relative fluorescence plotted along a 10-13 $\mu \mathrm{m}$ line bisecting the stained nucleoids. Bars, $4 \mu \mathrm{m}$. 
Table 3. Interactions between EsxA and EsxB detected using a bacterial two-hybrid system

Figures in parentheses are $\beta$-galactosidase units per milligram bacterial dry weight. - , No interaction; ++ , strong interaction.

\begin{tabular}{|lcccc|}
\hline \multirow{2}{*}{ Target } & \multicolumn{5}{c|}{ Bait } \\
\cline { 2 - 5 } & EsxA $_{\mathbf{M t}}$ & EsxA $_{\mathbf{S c}}$ & EsxB $_{\mathbf{M t}}$ & EsxB $_{\mathbf{S c}}$ \\
\hline EsxA $_{\mathrm{Mt}}$ & $-(125)$ & $-(131)$ & $++(6585)$ & $-(140)$ \\
EsxA $_{\mathrm{Sc}}$ & $-(136)$ & $-(113)$ & $-(108)$ & $++(7241)$ \\
EsxB $_{\mathrm{Mt}}$ & $++(5684)$ & $-(134)$ & $-(145)$ & $-(123)$ \\
EsxB $_{\mathrm{Sc}}$ & $-(151)$ & $++(7013)$ & $-(134)$ & $-(121)$ \\
\hline
\end{tabular}

The esx locus in $S$. coelicolor was originally predicted by comparative genome analysis with the esx clusters of $M$. tuberculosis (Gey Van Pittius et al., 2001). Indeed, it most closely resembles the es $x-4$ locus that phylogenetic analysis indicates to be the ancestral mycobacterial locus. The es $x-4$ locus also encodes the least number of proteins, with for example a single FtsK-SpoIIIE orthologue, EccC, rather than the function provided by the two smaller 'split' orthologues, EccCa and EccCb, encoded by the es $x-1$ locus, although no functional analysis of the $e s x-4$ genes has been reported. In S. coelicolor, secretion of both EsxA and EsxB substrates is dependent on the function of the predicted FtsK-SpoIIIE ATPase encoded by SCO5374 and the membrane-associated protein encoded by SCO5721 (an orthologue of $R v 3869$ ), but is independent of the function of either the serine protease encoded by SCO5722 or the integral membrane protein SCO5735 (an orthologue of Rv3877). Although we cannot exclude that other genes neighbouring or unlinked to the cluster may provide additional functions for the Streptomyces type VII secretion system, our initial analysis indicates that in terms of its complexity the machinery has fewer components compared with the mycobacterial ESX-1 system. In this respect it may be related to the simpler type VII secretion system of Staph. aureus, which requires the function of the FtsKSpoIIIE protein EssC and two smaller predicted membrane proteins, EssA and EssB, for secretion of the substrates EsxA and EsxB (Burts et al., 2005). Other genes in the $S$. aureus ess cluster, for example esaA, which encodes an integral membrane protein, are dispensable for secretion of the substrates.

In M. tuberculosis, an interaction between a specific Cterminal seven amino acid signal of EsxB and Rv3871 is necessary for secretion of the former (Champion et al., 2006). As secretion of EsxA is dependent on functional EsxB, a model for secretion of these substrates posits that
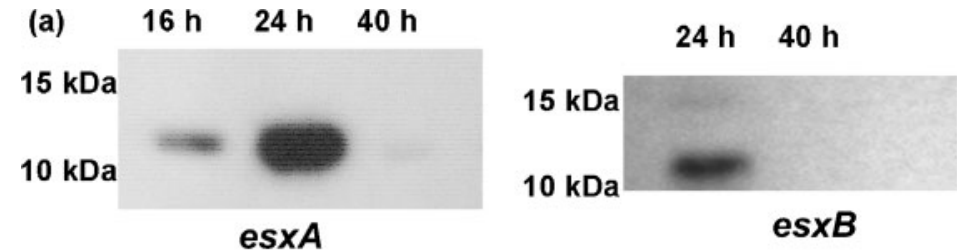

(b)

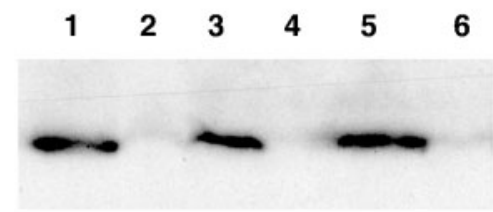

Extracellular

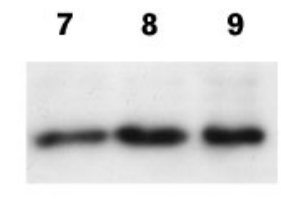

Intracellular

Fig. 4. Secretion of $E s x A_{[H i s] 6}$ and $E_{s x B} B_{[H i s] 6}$. (a) EsxA $_{[\text {His]6 }}$ (left-hand panel) or EsxB ${ }_{[\text {His]6 }}$ (righthand panel) were detected by immunoblotting in the supernatants of cultures of M145 containing pSAHis2 or pSAHis4 grown for the indicated time period (10 $\mu \mathrm{g}$ total protein per lane). (b, c) To investigate the dependence of $\mathrm{EsxA}_{[\mathrm{His}] 6}$ secretion on other functions encoded by the esx locus, strains containing pSAHis2 [except lane 2 in (b), with the empty vector $\mathrm{pSH} 152$ ] were grown for $24 \mathrm{~h}$ and the presence of $\mathrm{EsxA}_{[\mathrm{His}] 6}$ was analysed in both extracellular and intracellular fractions: (b) lane 1, M145, extracellular fraction; (b) lane 2, M145, extracellular fraction; (b) lane 3/ (c) lane 1, DSCO5724 (esxB::Tn5062), extracellular fraction; (b) lane 4, DSCO5734 (SC05734::Tn5062), extracellular fraction; (b) lane 5, DSCO5722 (SCO5722::Tn5062), extracellular fraction; (b) lane 6, DSCO5721 (SC05721::Tn5062), extracellular fraction; (b) lane 7, DSC05734 (SC05734::Tn5062), intracellular fraction; (b) lane 8, DSCO5722 (SC05722::Tn5062), intracellular fraction; (b) lane 9, DSC05721 (SC05721::Tn5062), intracellular fraction; (c) lane 2, DSCO5724 (esxB::Tn5062), intracellular fraction; (c) lane 3, DSCO5735 (SC05735::Tn5062), extracellular fraction; (c) lane 4, DSCO5735 (SC05735::Tn5062), intracellular fraction (5 $\mu \mathrm{g}$ total protein per lane). 


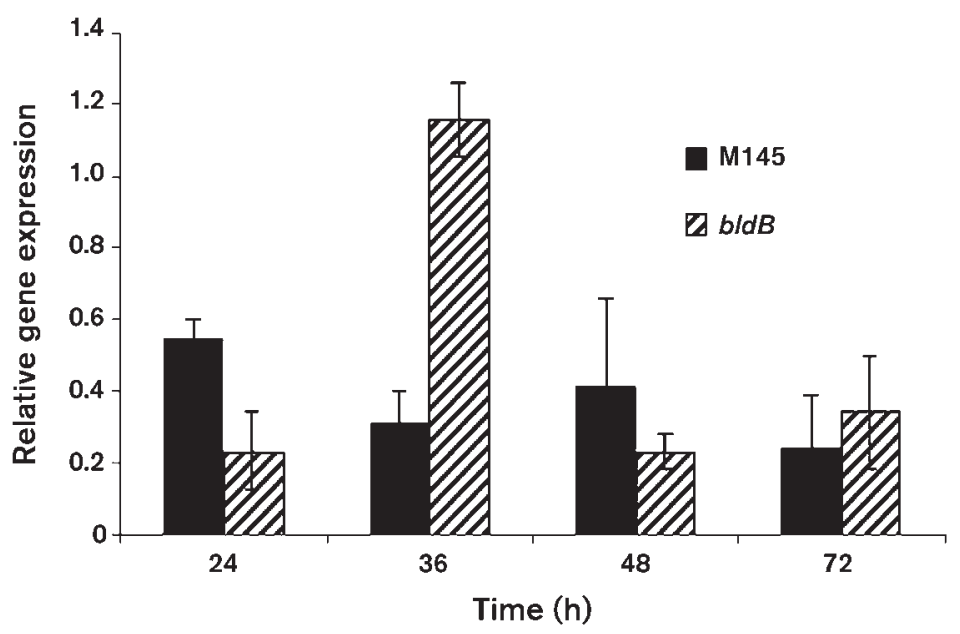

Fig. 5. Regulation of esxBA by BldB. Q RTPCR analysis of the abundance of es $B$ (SCO5724) mRNA in the parental strain M145 and a b/dB mutant, DSCO5723. The strains were grown on MS medium and RNA was purified at the times indicated. The data were normalized using $h r d B$ mRNA as a control. The data were obtained from two biological replicates with three technical replicates per biological replicate for each time point.

the cytoplasmic proteins form a stable heterodimer and that the heterodimer is targeted for secretion by interaction between the signal in the unstructured C-terminal domain of EsxB and the FtsK-SpoIIIE protein. Although our data indicate that $S$. coelicolor EsxA and EsxB likewise form a heterodimer, there is no obvious amino acid sequence homology at the $\mathrm{C}$ terminus of the $\mathrm{EsxB}$ protein with the signal sequence of the $M$. tuberculosis protein (Supplementary Fig. S2). Although this signal is conserved in other EsxB orthologues (e.g. Staph. aureus EsxB), it is also absent in mycobacterial paralogues such as EsxU encoded by the es $x-4$ locus. It remains to be determined how the $S$. coelicolor heterodimer is targeted for secretion.

Loss of function of the EsxAB heterodimer impacts on sporulation. The esxBA mutant has irregular-sized prespore compartments with corresponding aberrant DNA contents; there is an increased abundance of large compartments that apparently contain multiple nucleoids. This implies a loss of coordination of cell division with segregation of nucleoids. Orchestration of multiple synchronous cell division with compaction and segregation of individual nucleoids in aerial hyphal filaments is a complex albeit poorly understood process. Several functions have been identified that influence the outcome. The S. coelicolor SMC protein is associated with the nucleoid at specific foci during early stages of sporulation septation, and disruption of $s m c$ results in less-condensed nucleoids and a higher frequency of anucleate spore compartments (Kois et al., 2009). The defects in segregation are enhanced by combining smc, ftsK and parB mutations (Dedrick et al., 2009), confirming the functional interplay between nucleoid-condensing (SMC) and segregosome elements (FtsK, ParB). But even in a triple ftsK smc parB mutant, the majority of spores contain complete nucleoids, implying that coordination of nucleoid condensation and segregation with synchronous multiple septation in an aerial filament is dependent on several overlapping and partially redundant functions. Apparently the EsxAB heterodimer also has a fundamental role in the process, although the frequency of anucleate compartments produced by the mutants does not differ from the wild-type frequency (results not shown). The irregular-sized pre-spore compartments with corresponding aberrant DNA contents of the esxBA mutant resemble the phenotype of an $S$. coelicolor dpsA mutant (Facey et al., 2009). The Dps (DNA protection in starved cells) proteins in Streptomyces play an important role in the massive topological restructuring of nucleoids during sporulation, when the diffuse nucleoids of vegetative filaments become highly condensed to permit the subsequent segregation of one nucleoid per pre-spore compartment. We believe that the EsxAB heterodimer could regulate this process and propose a model whereby the heterodimer functions to sequester one or more factors that function to regulate correct nucleoid condensation. Due to the relatively low abundance of EsxAB (as estimated from Western blots), this factor is less likely to be an abundant structural protein associated with the nucleoid, and more likely to be a key regulator of expression of nucleoid-associated proteins. A precedent for an interaction between EsxAB and the regulatory protein EspR is provided in $M$. tuberculosis (Raghavan et al., 2008). EspR is a transcription factor that regulates expression of ESX-1 and is secreted by this system in an EsxAB-dependent manner. Sequestration of a regulatory protein that acts during vegetative growth as a brake to prevent nucleoid restructuring would represent a commitment to sporulation, and this would be reinforced by its subsequent secretion via the ESX system. SCO6675, an EspR orthologue in S. coelicolor, is a candidate regulator whose function we are currently investigating. Alternatively, BldB could be sequestered. This is a critical pleiotropic morphogenetic regulator linked to the ESX-1 locus in many sequenced streptomycetes; we have shown that the es $x B A$ operon is either directly or indirectly repressed by BldB during early sporulation. Interestingly, overexpression of $\mathrm{BldB}$, which could have an impact on es $x B A$ expression, has been shown to block sporulation after development of aerial hyphae, indicating that levels of this morphogen are critical for correct progression of the developmental programme (Pope et al., 1998). 
While the ESX-1 locus is the major virulence determinant of $M$. tuberculosis it also influences bacterial colony morphology. A change in colony morphology was observed in the initial passaging of $M$. bovis to obtain the BCG vaccine strain (Calmette, 1927). Complementation of BCG with RD1 DNA reverses this change to large extent and, similarly, addition of this region to an M. microti vaccine strain alters its colony morphology (Pym et al., 2002). For both these mycobacterial vaccine strains, the change in colony morphology has also been reported when only a subfragment of RD1 is introduced that is insufficient to restore all necessary functions of the type VII secretion system. The subfragment includes the esx $A B$ operon and two immediate upstream genes not considered critical components of the secretion system. This suggests that the intracellular EsxAB heterodimer influences mycobacterial cell structure, in addition to the virulence function of the secreted complex. This parallels our observations in $S$. coelicolor. An important intracellular morphogenetic function of the heterodimer in S. coelicolor is indicated by the difference between the delayed sporulation phenotype of es $x B A$ mutants and the normal sporulation exhibited by the secretion mutants. Clearly, this intracellular role in actinomycetes, possibly involving sequestration of one or more morphogenetic regulators as discussed, warrants further investigation.

\section{ACKNOWLEDGEMENTS}

This research has been supported by grants from the UK Biotechnology and Biological Sciences Research Council (BBSRC) (BB/E019242/1) and the European Commission (LSH-IP 005224 and ALFA II-01313-FA-FCD).

\section{REFERENCES}

Abdallah, A. M., Gey van Pittius, N. C., Champion, P. A., Cox, J., Luirink, J., Vandenbroucke-Grauls, C. M., Appelmelk, B. J. \& Bitter, W. (2007). Type VII secretion - mycobacteria show the way. Nat Rev Microbiol 5, 883-891.

Abramoff, M. D., Magelhaes, P. J. \& Ram, S. J. (2004). Image processing with ImageJ. Biophotonics International 11, 36-42.

Behr, M. A., Wilson, M. A., Gill, W. P., Salamon, H., Schoolnik, G. K., Rane, S. \& Small, P. M. (1999). Comparative genomics of BCG vaccines by whole-genome DNA microarray. Science 284, 1520-1523.

Bishop, A., Fielding, S., Dyson, P. \& Herron, P. (2004). Systematic insertional mutagenesis of a streptomycete genome: a link between osmoadaptation and antibiotic production. Genome Res 14, 893-900.

Bitter, W., Houben, E. N., Bottai, D., Brodin, P., Brown, E. J., Cox, J. S., Derbyshire, K., Fortune, S. M., Gao, L. Y. \& other authors (2009). Systematic genetic nomenclature for type VII secretion systems. PLoS Pathog 5, e1000507.

Brodin, P., Eiglmeier, K., Marmiesse, M., Billault, A., Garnier, T., Niemann, S., Cole, S. T. \& Brosch, R. (2002). Bacterial artificial chromosome-based comparative genomic analysis identifies Mycobacterium microti as a natural ESAT-6 deletion mutant. Infect Immun 70, 5568-5578.

Brodin, P., Majlessi, L., Marsollier, L., de Jonge, M. I., Bottai, D., Demangel, C., Hinds, J., Neyrolles, O., Butcher, P. D. \& other authors
(2006). Dissection of ESAT-6 system 1 of Mycobacterium tuberculosis and impact on immunogenicity and virulence. Infect Immun 74, 8898.

Burts, M. L., Williams, W. A., DeBord, K. \& Missiakas, D. M. (2005). EsxA and EsxB are secreted by an ESAT-6-like system that is required for the pathogenesis of Staphylococcus aureus infections. Proc Natl Acad Sci U S A 102, 1169-1174.

Burts, M. L., DeDent, A. C. \& Missiakas, D. M. (2008). EsaC substrate for the ESAT-6 secretion pathway and its role in persistent infections of Staphylococcus aureus. Mol Microbiol 69, 736-746.

Calmette, A. (1927). In La Vaccination Preventive Contre la Tuberculose, pp. 250. Paris: Masson et Cie.

Champion, P. A., Stanley, S. A., Champion, M. M., Brown, E. J. \& Cox, J. S. (2006). C-terminal signal sequence promotes virulence factor secretion in Mycobacterium tuberculosis. Science 313, 1632-1636.

Cole, S. T., Brosch, R., Parkhill, J., Garnier, T., Churcher, C., Harris, D., Gordon, S. V., Eiglmeier, K., Gas, S. \& other authors (1998). Deciphering the biology of Mycobacterium tuberculosis from the complete genome sequence. Nature 393, 537-544.

Dedrick, R. M., Wildschutte, H. \& McCormick, J. R. (2009). Genetic interactions of $s m c$, ftsK, and parB genes in Streptomyces coelicolor and their developmental genome segregation phenotypes. J Bacteriol 191, 320-332.

Del Sol, R., Mullins, J. G., Grantcharova, N., Flardh, K. \& Dyson, P. (2006). Influence of CrgA on assembly of the cell division protein FtsZ during development of Streptomyces coelicolor. J Bacteriol 188, 1540-1550.

Del Sol, R., Armstrong, I., Wright, C. \& Dyson, P. (2007). Characterization of changes to the cell surface during the life cycle of Streptomyces coelicolor: atomic force microscopy of living cells. J Bacteriol 189, 2219-2225.

Facey, P. D., Hitchings, M. D., Saavedra-Garcia, P., FernandezMartinez, L., Dyson, P. J. \& Del Sol, R. (2009). Streptomyces coelicolor Dps-like proteins: differential dual roles in response to stress during vegetative growth and in nucleoid condensation during reproductive cell division. Mol Microbiol 73, 1186-1202.

Fernandez-Martinez, L., Bishop, A., Parkes, L., Del Sol, R., Salerno, P., Sevcikova, B., Mazurakova, V., Kormanec, J. \& Dyson, P. (2009). Osmoregulation in Streptomyces coelicolor: modulation of SigB activity by OsaC. Mol Microbiol 71, 1250-1262.

Flardh, K. \& Buttner, M. J. (2009). Streptomyces morphogenetics: dissecting differentiation in a filamentous bacterium. Nat Rev Microbiol 7, 36-49.

Flett, F., Mersinias, V. \& Smith, C. P. (1997). High efficiency intergeneric conjugal transfer of plasmid DNA from Escherichia coli to methyl DNA-restricting streptomycetes. FEMS Microbiol Lett 155, 223-229.

Gey Van Pittius, N.C., Gamieldien, J., Hide, W., Brown, G.D., Siezen, R.J. \& Beyers, A.D. (2001). The ESAT-6 gene cluster of Mycobacterium tuberculosis and other high G+C Gram-positive bacteria Genome Biol 2, RESEARCH0044.

Guinn, K. M., Hickey, M. J., Mathur, S. K., Zakel, K. L., Grotzke, J. E., Lewinsohn, D. M., Smith, S. \& Sherman, D. R. (2004). Individual RD1-region genes are required for export of ESAT-6/CFP-10 and for virulence of Mycobacterium tuberculosis. Mol Microbiol 51, 359-370.

Hsu, T., Hingley-Wilson, S. M., Chen, B., Chen, M., Dai, A. Z., Morin, P. M., Marks, C. B., Padiyar, J., Goulding, C. \& other authors (2003). The primary mechanism of attenuation of bacillus Calmette-Guerin is a loss of secreted lytic function required for invasion of lung interstitial tissue. Proc Natl Acad Sci U S A 100, 12420-12425. 
Karimova, G., Pidoux, J., Ullmann, A. \& Ladant, D. (1998). A bacterial two-hybrid system based on a reconstituted signal transduction pathway. Proc Natl Acad Sci U S A 95, 5752-5756.

Kieser, T., Bibb, M. J., Buttner, M. J., Chater, K. F. \& Hopwood, D. A. (2000). Practical Streptomyces Genetics. Norwich, UK: John Innes Foundation.

Kois, A., Swiatek, M., Jakimowicz, D. \& Zakrzewska-Czerwinska, J. (2009). SMC protein-dependent chromosome condensation during aerial hyphal development in Streptomyces. J Bacteriol 191, 310-319.

Lightbody, K. L., Renshaw, P. S., Collins, M. L., Wright, R. L., Hunt, D. M., Gordon, S. V., Hewinson, R. G., Buxton, R. S., Williamson, R. A. \& Carr, M. D. (2004). Characterisation of complex formation between members of the Mycobacterium tuberculosis complex CFP-10/ESAT-6 protein family: towards an understanding of the rules governing complex formation and thereby functional flexibility. FEMS Microbiol Lett 238, 255-262.

Lightbody, K. L., Ilghari, D., Waters, L. C., Carey, G., Bailey, M. A., Williamson, R. A., Renshaw, P. S. \& Carr, M. D. (2008). Molecular features governing the stability and specificity of functional complex formation by Mycobacterium tuberculosis CFP-10/ESAT-6 family proteins. J Biol Chem 283, 17681-17690.

MacGurn, J. A. \& Cox, J. S. (2007). A genetic screen for Mycobacterium tuberculosis mutants defective for phagosome maturation arrest identifies components of the ESX-1 secretion system. Infect Immun 75, 2668-2678.

Mahairas, G. G., Sabo, P. J., Hickey, M. J., Singh, D. C. \& Stover, C. K. (1996). Molecular analysis of genetic differences between Mycobacterium bovis BCG and virulent M. bovis. J Bacteriol 178, 1274-1282.

Mishig-Ochiriin, T., Won, H. S., Lee, C. J., Kang, S. O. \& Lee, B. J. (2003). Biophysical and structural property of the putative DNAbinding protein, BldB, from Streptomyces lividans. Biopolymers 69, 343-350.

Mistry, B. V., Del Sol, R., Wright, C., Findlay, K. \& Dyson, P. (2008). FtsW is a dispensable cell division protein required for Z-ring stabilization during sporulation septation in Streptomyces coelicolor. J Bacteriol 190, 5555-5566.

Pallen, M. J. (2002). The ESAT-6/WXG100 superfamily - and a new Gram-positive secretion system? Trends Microbiol 10, 209-212.

Pope, M. K., Green, B. \& Westpheling, J. (1998). The bldB gene encodes a small protein required for morphogenesis, antibiotic production, and catabolite control in Streptomyces coelicolor. J Bacteriol 180, 1556-1562.

Pym, A. S., Brodin, P., Brosch, R., Huerre, M. \& Cole, S. T. (2002). Loss of RD1 contributed to the attenuation of the live tuberculosis vaccines Mycobacterium bovis BCG and Mycobacterium microti. Mol Microbiol 46, 709-717.

Raghavan, S., Manzanillo, P., Chan, K., Dovey, C. \& Cox, J. S. (2008). Secreted transcription factor controls Mycobacterium tuberculosis virulence. Nature 454, 717-721.

Renshaw, P. S., Lightbody, K. L., Veverka, V., Muskett, F. W., Kelly, G., Frenkiel, T. A., Gordon, S. V., Hewinson, R. G., Burke, B. \& other authors (2005). Structure and function of the complex formed by the tuberculosis virulence factors CFP-10 and ESAT-6. EMBO J 24, 24912498.

Sambrook, J., Fritsch, E. F. \& Maniatis, T. (1989). Molecular Cloning: a Laboratory Manual. Cold Spring Harbor, NY: Cold Spring Harbor Laboratory.

Schwedock, J., McCormick, J. R., Angert, E. R., Nodwell, J. R. \& Losick, R. (1997). Assembly of the cell division protein FtsZ into ladder-like structures in the aerial hyphae of Streptomyces coelicolor. Mol Microbiol 25, 847-858.

Simeone, R., Bottai, D. \& Brosch, R. (2009). ESX/type VII secretion systems and their role in host-pathogen interaction. Curr Opin Microbiol 12, 4-10.

Stanley, S. A., Raghavan, S., Hwang, W. W. \& Cox, J. S. (2003). Acute infection and macrophage subversion by Mycobacterium tuberculosis require a specialized secretion system. Proc Natl Acad Sci U S A 100, 13001-13006

Stanley, S. A., Johndrow, J. E., Manzanillo, P. \& Cox, J. S. (2007). The type I IFN response to infection with Mycobacterium tuberculosis requires ESX-1-mediated secretion and contributes to pathogenesis. J Immunol 178, 3143-3152.

Volkman, H. E., Clay, H., Beery, D., Chang, J. C., Sherman, D. R. \& Ramakrishnan, L. (2004). Tuberculous granuloma formation is enhanced by a mycobacterium virulence determinant. PLoS Biol 2, e367.

Yanisch-Perron, C., Vieira, J. \& Messing, J. (1985). Improved M13 phage cloning vectors and host strains: nucleotide sequences of the M13mp18 and pUC19 vectors. Gene 33, 103-119.

Edited by: M. S. Paget 